\title{
Examinando las redes de actores en el análisis de las políticas públicas: debate teórico y técnicas cuantitativas
}

Jacint Jordana

Coordinador del número

Analizar los actores que participan en la definición, elaboración o puesta en práctica de las políticas públicas es una tarea muy importante, por cuanto nos permite conocer a los protagonistas del proceso político así como avanzar en el conocimiento de los motivos de sus acciones. Las limitaciones de carácter interno que afrontan para desarrollar sus estrategias y conseguir sus objetivos, las relaciones que establecen entre ellos y el estilo a la hora de configurar sus posiciones son algunos de los aspectos que pueden ser explorados y discutidos de forma sistemática con el apoyo de distintos modelos interpretativos. El análisis de las redes de actores constituye una de las posibles direcciones a seguir para estudiar el papel de los actores en los procesos políticos, tomando sin duda una perspectiva de carácter más estructural, en el sentido de suponer que la configuración de las relaciones entre los actores nos puede ayudar a entener el proceso político, más alla de las caracteristicas puramente individuales de cada uno de los participantes.

Así, partiendo de esta concepción, el estudio de las redes de actores en el marco del análisis de las políticas públicas sectoriales, y más en general sobre el proceso de toma de decisiones políticas, se ha ido desarrollando paulatinamente en las últimas décadas (Rhodes, 1990; Marin y Mayntz, 1991; Jordana, 1995; Thatcher, 1998), y actualmente constituye una perspectiva con una trayectoria ya ciertamente prolongada dentro de la Ciencia Política, que ha dado lugar a un amplio número de estudios y publicaciones, bien de carácter teórico, bien empírico, en las que se han empleado herramientas cuantitativas y cualitativas (Leifeld, 2007; Schneider et al., 2007).

Aunque los estudios basados en aplicaciones del análisis de redes de actores han sido frecuentes a lo largo de los años, también es cierto que a menudo han ido acompañados de una intensa discusión conceptual sobre la potencialidad de este enfoque para esclarecer algunos de los problemas clásicos del análisis de las políticas públicas. Tal vez, como señala Borzel (1998), una forma de simplificar la confusa variedad de interpretaciones y aplicaciones vinculadas con las redes de políticas públicas sea considerar la exis- 
tencia de dos grandes visiones entorno a esta perspectiva. Por un lado, la escuela anglosajona, que vincula el estudio de las redes con los modelos de intermediación de intereses y, en un plano más empírico, con la aproximación cuantitativa a las interpretaciones pluralistas; y por otro lado, la escuela germánica, más cercana a los modelos teóricos de la gobernanza, donde las redes se equiparan con formas de dirección política distintas a las jerarquías y los mercados, identificando a éstas con la infraestructura de procesos más consensuales de toma de decisiones. Estas dos visiones han dado lugar a una gran variedad de conceptos e interpretaciones, incluyendo algunas aproximaciones híbridas (van Waarden, 1992; Rhodes, 1997), así como numerosas polémicas y discusiones, que a veces ponían en duda su propio valor explicativo como perspectiva teórica (Dowding, 1995) o criticaban la relativa ausencia del Estado en su análisis (Zurbriggen, 2004).

En la última década, no obstante, la intensidad de los debates más teóricos se ha ido reduciendo, especialmente en lo que se refiere a las expectativas de poder explicar, mediante la identificación de la estructura de las redes, los posibles resultados de las políticas y las formas que podrían tomar los procesos políticos. El determinismo inherente en estas aspiraciones se ha visto escasamente refrendado por resultados empíricos, mientras que se ha destacado más las posibilidades de análisis de contexto que ofrece el estudio de las redes de actores, como por ejemplo la posibilidad de identificar distintas configuraciones de poder a través de sectores y países (Adam y Kriesi, 2007), o la capacidad de determinadas estructuras relacionales para reducir los costes de transacción en los procesos de decisión política (Hindmoor, 1998). Estudios recientes con una fuerte orientación empírica, utilizando diversos métodos cuantitativos para medir la estructura de las redes, nos muestran algunas aplicaciones del análisis de redes de actores que incluyen las lógicas de gobierno multi-nivel en las configuraciones de poder (Kriesi, Adam y Jochum, 2006) o la evolución de las redes de asociaciones empresariales frente a los cambios de su entorno (Grote, Lang y Schneider, 2008).

El análisis empírico de las redes de actores nos permite identificar la percepción de cada actor sobre el resto y su capacidad de influencia a la hora de tomar las decisiones que afectan al sector. Ello nos conduce a la formación de una matriz sobre representaciones de poder dentro de las redes y a una valorización de las interacciones entre los actores. Esta percepción mutua del poder entre los actores pertenecientes a una red de políticas es una visión subjetiva, esto es, de cada actor respecto a los demás. Sin embargo, las valoraciones subjetivas están en la base de la aceptación de determinadas decisiones claves, y pueden además llegar a ser expresión de unas reglas informales que el resto de actores acaten (el poder de quien las impone -por su capacidad de penalización, aunque raramente se ejerza- obliga a su cumplimiento). En este sentido, para observar la configuración del poder, es más productivo explorar las valoraciones subjetivas que las manifestaciones efectivas -las cuales sólo aparecen ocasionalmente en algunas coyunturas críticas-, analizando las estructuras de relación que se forman entre ellas¹. Luego la utilización de tipos ideales relativos a

1 Para una aplicación al caso de las políticas de telecomunicaciones en España en el contexto de la liberación del sector, véase Jordana y Sancho (2005). 
distintas formas de gobernanza, entendidos como formas de configuración del poder en un espacio político acotado sectorialmente, constituye una vía para articular interpretaciones más complejas de la variedad de procesos políticos.

Por otra parte, las redes de actores también se pueden analizar cuantitativamente desde el punto de vista de sus relaciones objetivas no basadas en percepciones sino en intercambios de información, contactos personales, elementos compartidos, etc. que sirven como indicadores de la proximidad entre los actores y del tipo de relación que establecen, y que pueden ser medidos con precisión recogiendo información detallada mediante encuestas u otras fuentes de información (por ejemplo, flujos de comunicación o encuentros presenciales). Las formas estructurales identificadas a partir de estos datos pueden analizarse con precisión, identificando los espacios de mayor densidad de relaciones en la red, la centralidad de determinados actores y muchas otras características específicas -como el papel de las reglas formales de decisión, por ejemplo- que permiten una detallada comprensión de las capacidades de los actores para impulsar y ajustar sus preferencias sobre las políticas públicas en las interacciones que establecen ${ }^{2}$. Los resultados obtenidos en este tipo de análisis no permiten inferencias deterministas sobre los resultados de las políticas, pero sí aportan unas explicaciones contextuales de gran utilidad para la interpretación de las posiciones y estrategias de los actores en el proceso político, y pueden contribuir a clarificar enormemente los análisis de interacciones críticas, que por la necesaria claridad de los modelos, a menudo se encuentran excesivamente simplificados.

Sin duda, las posiblemente exageradas expectativas teóricas planteadas por el análisis de las redes de políticas públicas, en el sentido de llegar a generar una teoría de éstas como forma de gobernanza, o concebir de forma determinista que sus estructuras podrían tener una cierta capacidad explicativa sobre los resultados de las políticas, no se han visto confirmadas en modo alguno; sin embargo, de tales aspiraciones están surgiendo nuevas formas de análisis del papel de los actores en las políticas públicas. Así, la capacidad de medir con precisión la estructura de las redes que forman los actores de las políticas públicas permite avanzar en la discusión de hipótesis más concretas relativas al papel de los contextos estructurales sobre los procesos de decisión, tanto los que se refieren a las percepciones y las reglas informales sobre el poder, como los que identifican los canales y formas de relación entre actores y su articulación dentro de las redes que forman. El futuro del análisis de las redes de actores en las políticas públicas depende en buena medida de cómo vayan surgiendo diseños de investigación más complejos que, con la ayuda de las técnicas del análisis de redes sociales, permitan explorar problemas más sofisticados de la relación entre estructura y acción en los procesos políticos.

Este número monográfico de la revista constituye una modesta aportación en la dirección arriba mencionada, con la inclusión de cinco estudios empíricos que

2 Véase un detallado ejemplo sobre la estructura de relaciones entre las organizaciones del sector agropecuario en Santa Cruz, Bolivia (Porras Martínez, 2001). 
muestran distintas posibilidades de aplicación de métodos cuantitativos al análisis de las redes de políticas públicas, al tiempo que en sus diseños de investigación mantienen una conexión específica con alguno de los problemas teóricos ya señalados. Así, los autores plantean interpretaciones sobre las configuraciones que identifican en las redes de actores, más allá de la simple descripción factual, en diálogo con debates actuales sobre los procesos políticos y la articulación de intereses en las políticas públicas. Una característica adicional de este número monográfico es la discusión de temas vinculados con la realidad de las políticas públicas en España. Casi todos los trabajos, con una excepción, analizan temas relativos al caso español, bien tratado en su conjunto, bien comparando diversas comunidades autónomas. Por este motivo, sin duda este número monográfico constituye también una aportación al conocimiento específico de nuestra realidad política más cercana, lamentablemente aún poco explorada en lo que se refiere a los procesos de formación de sus políticas públicas.

En primer lugar, el artículo de Susana Borrás analiza el papel de la Comisión Europea como intermediario político en la tupida red de actores que configura las políticas públicas de dimensión europea en Bruselas. Su argumento consiste en mostrar que la Comisión es un actor central en estas redes, con una fuerte capacidad de intermediación en relación al resto de actores. Para ilustrar esta tesis, toma como caso de estudio dos sectores de políticas distintos, la estrategia de empleo europea, y la regulación de los organismos modificados genéticamente. Utilizando dos medidas distintas del papel de los actores en la estructura de las redes, su centralidad y su capacidad de intermediación, discute algunas hipótesis concretas sobre la capacidad de la Comisión Europea de mantener su papel como actor central para intermediar en diversas políticas públicas. Sus resultados muestran una clara visión de la estructura de relaciones en las redes de actores de las políticas europeas, y le permiten analizar los papeles sistemáticos que juegan los actores más prominentes.

A continuación, el artículo de Achim Lang, centrado en el ámbito español, aborda el tema de las organizaciones de intereses en el sector de las tecnologías de la información y la comunicación. Su propósito es entender cómo se ha desarrollado el mapa de asociaciones empresariales en las últimas décadas, identificando diversos patrones de evolución y diferenciación organizativa. El marco teórico empleado -sobre los procesos de evolución de las organizaciones de intereses- resulta innovador, lo que no impide que el empleo de conceptualizaciones clásicas como son las vinculadas a los modelos de carácter neo-corporativo sobre la representación de intereses. El artículo analiza la estructura de relaciones entre las diversas asociaciones identificadas, mostrando con precisión los diversos ejes de cooperación y conflicto que se encuentran presentes. Para esta finalidad, Lang se apoya en las técnicas de análisis de las redes sociales, lo que le permite medir las pautas de relación establecidas entre estas organizaciones. Es precisamente la aplicación clara y sistemática de estas técnicas lo que permite al autor discutir con exactitud sus hipótesis de trabajo y confirmar la adecuación del marco teórico empleado para explicar la representación de intere-

12 ses empresariales en este sector. 
También el trabajo de Laura Chaqués se centra principalmente, aunque no exclusivamente, en el nivel estatal, analizando la evolución reciente de la política farmacéutica en España. Su estudio discute el cambio de la política, la transformación de la estructura de actores y sus relaciones a lo largo de la última década. El estudio se centra en la influencia de las transformaciones institucionales experimentadas en este sector sobre la estructura de la red de actores en perspectiva multinivel, desde la influencia creciente de la política y las instituciones europeas en el sector, hasta el impacto del Estado de las autonomías. Así, la reconfiguración de la red de actores hacia un modelo más fragmentado se relaciona con la emergencia de nuevas instituciones que ocupan posiciones claves y transforman la propia estructura de la red. En este sentido, la interpretación teórica que aporta el estudio de Laura Chaqués, vinculando el estudio de las redes de políticas públicas con diversos modelos más generales de análisis de las políticas públicas, nos abre de forma sugerente un marco para el debate sobre qué puede aportar el análisis de redes a la interpretación de los procesos de cambio de políticas.

Por su parte, Jacint Jordana, Fabiola Motta y Andrea Noferini realizan un análisis de la implementación de las políticas de cohesión europea en dos comunidades autónomas españolas. Centrándose en el examen de la estructura de las redes de actores vinculados a esta política e identificando aspectos tales como la reputación, la centralidad de los actores o la estructura de relaciones informales que se establecen entre ellos, el propósito del artículo es el de observar las diferencias que se producen en un espacio institucional casi idéntico a la hora de implementar las políticas de cohesión europea, espacio éste que viene definido por los requisitos formales establecidos por la Comisión Europea y por el propio marco político español. Las variaciones y similitudes de las redes identificadas se relacionan con las características del capital social de las elites políticas en cada región, mostrándose la existencia de distintos patrones de elaboración de políticas. Un marco conceptual que identifica distintos tipos ideales de procesos de intermediación de intereses sirve a los autores para discutir y clarificar las diferencias observadas, planteado el debate en una perspectiva comparada de carácter más general.

Finalmente, el trabajo de Mentxu Ramillo estudia las redes de actores implicados en un sector de políticas públicas emergente y con unos perfiles poco definidos, como son las políticas para impulsar la Sociedad de la Información, con el propósito de observar la formación de tales redes, generando espacios comunes donde se definían e impulsaban políticas públicas determinadas. Su trabajo compara la articulación de los diversos tipos de actores en dos comunidades autónomas distintas, Cataluña y el País Vasco, con el fin de analizar las diferencias existentes y discutir las posibles causas de las variaciones observadas. Las técnicas utilizadas para este propósito también se basan en el análisis de las redes sociales, aunque en este caso identificando tipos de actores muy variados, tanto públicos como privados. La autora aplica diversas medidas para analizar la estructura de relaciones entre los actores, lo que le permite identificar múltiples dimensiones subyacentes a tal estructura: las percepciones sobre la influencia de los actores, los contactos directos entre ellos, y las temáticas en 
torno a las que articulan sus relaciones. La comparación de los resultados obtenidos le permite identificar con precisión las importantes diferencias existentes entre las redes de actores de ambas comunidades autónomas, y apuntar su influencia sobre las políticas públicas llevadas a cabo en cada caso.

Aunque no es un tema central de ninguno de estos estudios, la perspectiva del gobierno multi-nivel se encuentra presente en casi todos ellos, al considerar que actores pertenecientes a otros niveles tiene una influencia importante en arenas políticas de niveles superiores o inferiores. Estos actores, y la discusión sobre su importancia, aparecen claramente identificados, y la estructura de los vínculos entre niveles de gobierno surge de forma patente, más allá de consideraciones generales, concretándose en actores específicos, que están presentes en diversos niveles al mismo tiempo. En su conjunto, por tanto, estos estudios apuntan también elementos sobre el funcionamiento efectivo del gobierno multi-nivel en España, desde una perspectiva empírica que destaca por la identificación de numerosas variaciones entre territorios, niveles y sectores. Sin duda, es ya el momento de dejar paso a los artículos, pero no sin antes agradecer a los colaboradores en este número monográfico su paciencia y confianza para compartir conmigo su tiempo y energía con el propósito de hacer realidad este proyecto común, combinando posiciones teóricas y métodos cuantitativos en el análisis de las redes de políticas públicas.

\section{REFERENCIAS BIBLIOGRÁFICAS}

Adam, Silke y Kriesi, Hans-Peter (2007), “The Network Approach”, en P. Sabatier, ed., Theories of the Policy Process, Westview Boulder.

Borzel, T. (1998), “Organizing Babylon - on the different conceptions of policy networks”, Public Administration, 46: 253-73.

Dowding, K. (1995), “Model or Metaphor? A Critical Review of the Policy Network Approach", Political Studies, 43: 136-158.

Grote, J.R., Lang. A. y Schneider, V. (eds) (2008), Organized interests in Changing Environments. The complexity of adaptation, Houndmills: Palgrave.

Hindmoor, A (1998), "The importance of being trusted: transaction costs and policy network theory”, Public Administration, 46: 25-43.

Jordana, J. (1995), “El análisis de los policy networks: ¿Una nueva perspectiva sobre la relación entre políticas públicas y Estado?”, Gestión y Análisis de Políticas Públicas, 3: 77-90.

Jordana, J. y Sancho, D. (2005), “Policy Networks and Market Opening: Telecommunications Liberalization in Spain”, European Journal of Political Research, 44 (4): 1-29. 
Kriesi, H.-P., Adam, S. y Margit, J. (2006), "Comparative analysis of policy networks in Western Europe”, Journal of European Public Policy, 13 (3): 341-361.

Leifeld, P. (2007), Policy Networks: A Citation Analysis of the Quantitative Literatutre, Diplomarbeit, Department of Politics and Management, University of Konstanz.

<http:www.ub.uni-konstanz.de/kops/volltexte/2007/2663.>

Marin, B. y Mayntz, R. (1991), Policy Networks: Empirical Evidence and Theoretical Considerations. Frankfurt: Campus Verlag.

Porras Martínez, J.I. (2001), "Policy Networks o redes de políticas públicas: una introducción a su metodología de investigación”, Estudios Sociológicos 19 (57): 721745 .

Rhodes, R.A.W. (1990), "Policy networks. A British perspective", Journal of Theoretical Politics, 2(3): 293-317.

Rhodes, R.A.W. (1997), Understanding Governance. Policy Networks, governance, reflexivity and accountability. Buckingham: Open University Press.

Schneider, V., Lang, A., Leifeld, P. y Gundelch, B. (2007), "Political Networks - A Structured Bibliography”. Universität Konstanz.

<www.polnet-school.info/download/PolNetw_StructBibliography.pdf>

Thatcher, M. (1998), “The Development of Policy Network Analyses: From Modest Origins to Overarching Frameworks", Journal of Theoretical Politics, 10 (4): 389416.

van Waarden, F. (1992), “Dimensions and Types of Policy Networks”, European Journal of Political Research, 21: 29-52.

Zurbriggen, C. (2004), "Redes, actores e instituciones", Revista del CLAD. Reforma y Democracia, 30: 167-188. 